\title{
Genome Sequencing of a Camelpox Vaccine Reveals Close Similarity to Modified Vaccinia virus Ankara (MVA)
}

\author{
Maurilia Marcacci ${ }^{1,2, *,+}$ (), Abdelmalik I. Khalafalla ${ }^{3,+}$, Zulaikha M. Al Hammadi ${ }^{3}$, \\ Federica Monaco ${ }^{1}$, Cesare Cammà ${ }^{1} \mathbb{D}$, Mohammed F. Yusof ${ }^{3}$, Saeed M. Al Yammahi ${ }^{3}$, \\ Iolanda Mangone ${ }^{1}$, Fabrizia Valleriani ${ }^{1}$, Mohamed A. Alhosani ${ }^{3}$, Nicola Decaro ${ }^{2} \mathbb{1}$, \\ Alessio Lorusso $^{1}$ (D), Salama S. Almuhairi ${ }^{3}$ and Giovanni Savini ${ }^{1}$ \\ 1 Istituto Zooprofilattico Sperimentale dell'Abruzzo e Molise, 64100 Teramo, Italy; f.monaco@izs.it (F.M.); \\ c.camma@izs.it (C.C.); i.mangone@izs.it (I.M.); f.valleriani@izs.it (F.V.); a.lorusso@izs.it (A.L.); \\ g.savini@izs.it (G.S.) \\ 2 Department of Veterinary Medicine, University of Bari, Valenzano, 70010 Bari, Italy; nicola.decaro@uniba.it \\ 3 Veterinary Laboratories Division, Abu Dhabi Agriculture and Food Safety Authority (ADAFSA), Abu Dhabi \\ 52150, UAE; abdelmalik.khalafalla@ADAFSA.GOV.AE (A.I.K.); \\ zulaikha.alhammadi@ADAFSA.GOV.AE (Z.M.A.H.); Mohd.Yusof@ADAFSA.GOV.AE (M.F.Y.); \\ saeed.alyammahi@ADAFSA.GOV.AE (S.M.A.Y.); mohamed.a.alhosani@ADAFSA.GOV.AE (M.A.A.); \\ salama.almuhairi@ADAFSA.GOV.AE (S.S.A.) \\ * Correspondence: m.marcacci@izs.it \\ $\dagger$ These authors contributed equally to this work.
}

Received: 6 June 2020; Accepted: 21 July 2020; Published: 23 July 2020

\begin{abstract}
Camelpox is a viral contagious disease of Old-World camelids sustained by Camelpox virus (CMLV). The disease is characterized by mild, local skin or severe systemic infections and may have a major economic impact due to significant losses in terms of morbidity and mortality, weight loss, and low milk yield. Prevention of camelpox is performed by vaccination. In this study, we investigated the composition of a CMLV-based, live-attenuated commercial vaccine using next-generation sequencing (NGS) technology. The results of this analysis revealed genomic sequences of Modified Vaccinia virus Ankara (MVA).
\end{abstract}

Keywords: camelpox; vaccine; Vaccinia virus; next-generation sequencing

\section{Introduction}

Camelpox is a viral contagious disease of Old-World camelids and is characterized by mild, local skin or severe systemic infections. This disease may have a major economic impact due to significant losses in terms of morbidity and mortality, weight loss, and low milk yield [1]. The causative agent is Camelpox virus (CMLV), a double-stranded (ds) DNA virus belonging to the genus Orthopoxvirus (OPXV) of the family Poxviridae, subfamily Chordopoxvirinae. Twelve species are recognized within this genus, and the most notable include Variola major virus (VARV), Vaccinia virus (VACV), and Cowpox virus (CPXV). The Orthopoxvirus genome is composed of several structures. Two inverted terminal repeats (ITRs), identical in sequence, are present at the ends of the dsDNA genome and form covalently closed hairpin structures [2]. In addition, ITRs consist of further sequence elements, including repeat and coding regions. Furthermore, left and right terminal regions flank a central conserved region. Left and right terminal regions contain open reading frames (ORFs) encoding for proteins involved in host interactions (host range, immunomodulation, and pathogenicity), while the central region encloses ORFs encoding for proteins involved in essential viral replicative processes [3]. However, 
differences in the genomes could be observed between the viral species of this genus. First, genome size may vary. Taking into account VACV, CPXV, and CMLV, it ranges from 194,000 (VACV) to 229,000 $(\mathrm{CPXV})$. Accordingly, genome constellations are also variable. As an example, genomic differences between CMLV and other OPXVs are greater in terminal regions due to small and large nucleotide (nt) insertions, deletions, and translocations [4]. Such deletions may also be due to cell-culture adaption. For instance, Modified Vaccinia virus Ankara (MVA), used during the last years of the WHO smallpox eradication campaign, shows a significantly smaller genome compared to extant VACV strains likely as a consequence of the massive passaging (over 570 times) in chicken embryo fibroblasts [5]. While other OPXVs can infect various hosts, CMLV seems to be restricted to a single host, and camels usually get infected by contact with infected animals through skin abrasions or via aerosol [1]. The virus can also spread in the environment either in water or on fomites [6,7]. Unlike other OPXVs, whose genome sequences are highly represented in public databases, the number of CMLV sequences is still very limited. Prevention and control of camelpox can be achieved using live-attenuated and inactivated vaccines. Recently, it was discovered that an attenuated CMLV vaccine, prepared and commercialized in the Kingdom of Saudi Arabia (KSA) using strain Jouf-78, contained VACV rather than CMLV [8]. In this study, by employing modern NGS technology, we attempted to determine and analyze the whole genome sequence (WGS) of another commercially available CMLV vaccine, the Ducapox vaccine.

\section{Materials and Methods}

\subsection{Ducapox Vaccine}

The live-attenuated Ducapox vaccine (batch number DPV0816, manufacture date August 2016, expiration date August 2017) contains $10^{6.5} \mathrm{TCID}_{50} / \mathrm{mL}$ of strain CaPV298-2, which was isolated in the United Arab Emirates (UAE) and attenuated by 120 serial passages into Vero cells [9]. The vaccine, originally developed by Onderstepoort Biological Products $[10,11]$ and produced by Highveld Biologicals, Republic of South Africa (RSA), is currently manufactured by the Central Veterinary Research Laboratory (CVRL, Dubai, UAE). A total volume of $11 \mathrm{~mL}$ of sterile, distilled water has been used for the reconstitution.

\subsection{NextSeq500 (Illumina) Sequencing}

DNA purification was performed starting from $200 \mu \mathrm{L}$ of reconstituted sample using the High Pure Viral Nucleic Acid Kit (Roche, Basel CH, Switzerland) according to the manufacturer's guidelines. DNA was then quantified by Qubit dsDNA HS assay (Thermo Fisher Scientific, Waltham, MA, USA), and one nanogram was used for library preparation by Nextera XT DNA Library Prep kit (Illumina Inc., San Diego, CA, USA) according to the manufacturer's protocol. Deep sequencing was performed on the NextSeq 500 platform (Illumina Inc., San Diego, CA, USA) using the NextSeq 500/550 Mid Output Reagent Cartridge v2, with 300 cycles and standard 150 bp paired-end reads [12].

\subsection{Phylogenetic Analysis}

Phylogenetic analysis was performed based on the nine concatenated open reading frames (ORFs) $[13,14]$, namely the early transcription factor/VETF enzyme large subunit (A7L), the major core protein (A10L), the RNA polymerase 132 (A24R), the messenger RNA capping enzyme the large subunit (D1R), the DNA-independent NTPase (DNA replication, D5R), the hypothetical protein (E6R9), the DNA polymerase (E9L), the RNA polymerase-associated protein (H4L), and the RNA polymerase 147 (J6R). Gene designations refer to the VACV-COP genome (GenBank accession No. M35027). The sequence dataset consisted of concatenated genome sequences of different OPXV species representative of North America and the Old World (Africa and Eurasia) that were used for alignment by Geneious version 10.1.3 and MAFFT algorithm [15]. Phylogenetic analysis was performed with Bayesian inference by using four chains run for $>1$ million generations [16,17]. Posterior output of the tree was derived from 
Bayesian inference using four chains run for $>1$ million generations, a general time-reversible model, a proportion of invariable sites, a gamma distribution of rate variation across sites, and a subsampling frequency of 1000. ModelTest software (http://evomics.org/resources/software/molecular-evolutionsoftware/modeltest/) was used to identify the most appropriate model of evolution for the entire dataset and for each gene individually. The identified program settings for all partitions, under the Akaike Information Criteria, included six character states (general time-reversible model), a proportion of invariable sites, and a gamma distribution of rate variation across sites.

\section{Results}

\subsection{Sequencing Revealed High Nucleotide Sequence Identity to Vaccinia Virus}

The sequencing run of Ducapox vaccine produced a total number of 1,202,012 reads. Quality controls and trimming carried out by FASTQC (v0.11.5) and trimmomatic-0.36 [18,19], maintained 969,312 reads with an average length of $91.7 \mathrm{nt}$ and mean quality of 30.73. Host depletion was performed by Bowtie 2 (v2.1.0) [20], and the remaining 74,876 reads (mean length 86.33 and mean quality 30.45) were used for de novo assembly by SPAdes software v. 3.11.1 [21,22] that produced a unique contig of 152,596 nt. BLASTn analysis was performed to identify the closest publicly available sequence in the GenBank database. The best match (99.99\%) was with the Vaccinia virus strain Acambis 3000 Modified Virus Ankara (accession No. AY603355), and this sequence was used to perform a reference-based assembly by Bowtie 2 (v2.1.0) [20]. This task produced a unique consensus sequence of $166,722 \mathrm{nt}$ with horizontal coverage of $96 \%$, as two gaps of $5195 \mathrm{nt}$ and $890 \mathrm{nt}$ were present. Deep mean coverage was 26X. The consensus sequence was annotated using Prokka v1.12 [23] and deposited with the GenBank database (MT648498). Nt sequence identity with the five CMLV whole genome sequences available on GenBank ranges from $97.54 \%$ to $97.56 \%$.

\subsection{Ducapox Vaccine Clusters with Vaccinia Virus Strains}

The nine ORFs (A7L, A10L, A24R, D1R, D5R, E6R, E9L, H4L, and J6R) of the Ducapox vaccine sequence were concatenated and aligned with homologous sequences of selected OPXV strains. Phylogenetic analysis was performed using Bayesian inference. Posterior probability percentages were consistently high ( $>95 \%)$ for all clades on phylograms, which supported inferred phylogenetic relationships.

In the phylogenetic tree (Figure 1), Ducapox vaccine was distantly related to CMLV strains; conversely, it showed a closer relatedness with VACV strains, albeit forming a separate subcluster with Vaccinia virus strain Acambis 3000 Modified Virus Ankara (MVA, AY603355) and Vaccinia virus strain Ankara (U94848) strains. Nt identity of Ducapox vaccine strain with different VACV strains ranged between 98.2 and $99.9 \%$. 


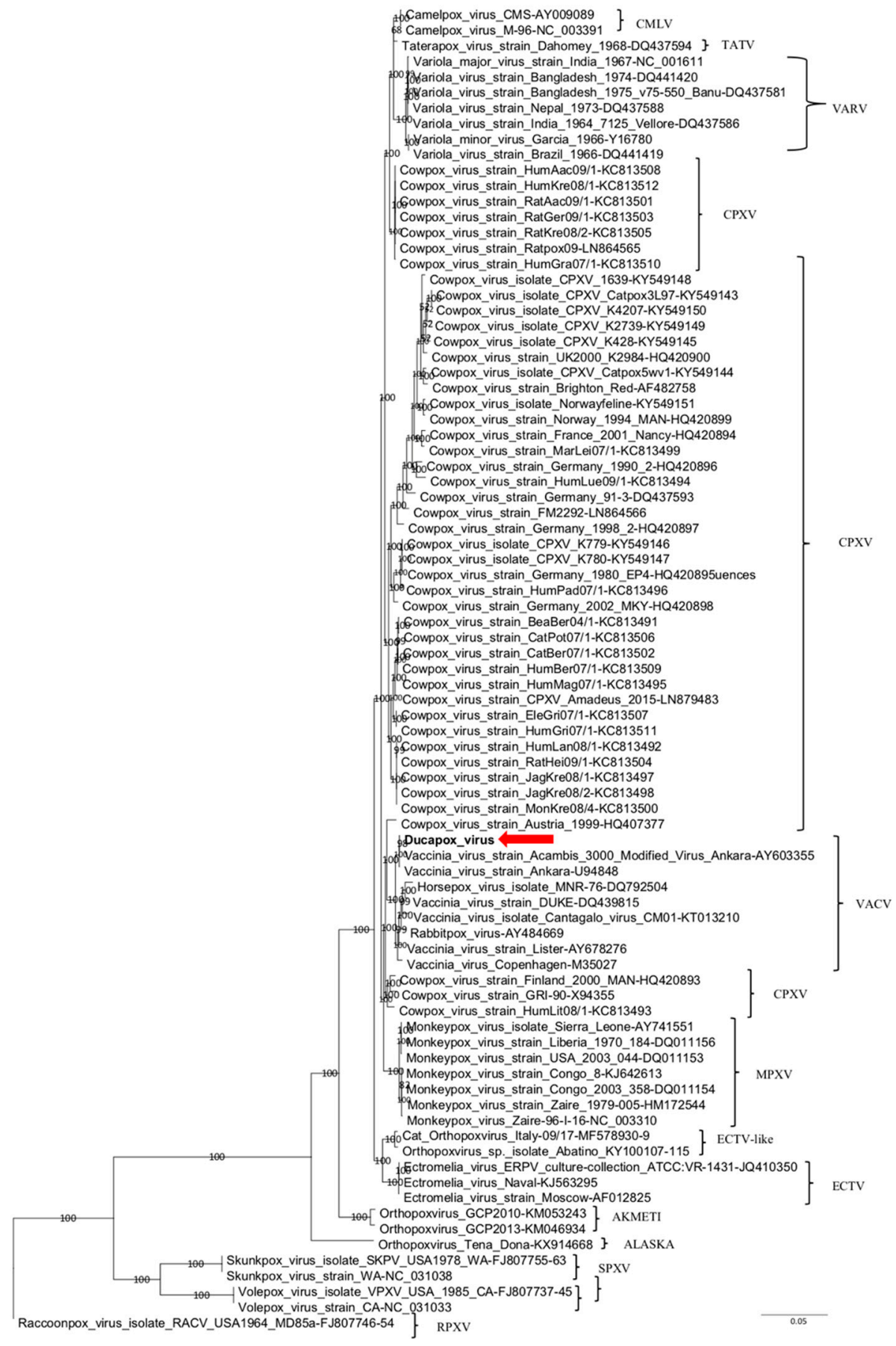

Figure 1. Phylogenetic relationship of extant OPXVs with Ducapox vaccine. Phylogenetic tree shows concatenated alignment of nine coding gene (A7L, A10L, A24R, D1R, D5R, E6R, E9L, H4L, and J6R) sequences of Orthopoxvirus. Gene designations refer to the VACV-COP genome (GenBank accession No. M35027). Posterior probability values $>0.95$ are indicated on the tree nodes. The red arrow indicates the Ducapox vaccine (this study). Raccoonpox virus strain MD85A was used as an outgroup. Scale bar indicates nucleotide substitutions per site. CMLV, Camelpox virus; CPXV, Cowpox virus; ECTV, Ectromelia virus; MPXV, Monkeypox virus; RPXV, Raccoonpox virus; SPXV, Skunkpox virus; TATV, Taterapox virus; VACV, Vaccinia virus; VARV, Variola virus; VPXV, Volepox virus. 


\section{Discussion}

In this study, the genome composition of Ducapox vaccine, a commercial CMLV vaccine produced by the Central Veterinary Research Laboratory (CVRL, Dubai, UAE) starting from the CMLV CaPV298-2 field strain, was investigated by NGS. The vaccine sequence content revealed the highest nt identity with an MVA strain (AY603355) of VACV and the absence of CMLV-unique regions including ORFs CMLV185, CMLV186, and CMLV187 [4]. Interestingly, nt sequences closely related to VACV strains were also revealed by Yousif and colleagues while investigating the genome composition of an additional commercially available, live-attenuated CMLV vaccine (Veterinary Vaccine Production Center (V.V.P.C.) Modified live virus vaccine for camelpox) based on the Jouf-78 field strain [8]. The only reasonable scenario explaining this bizarre situation is that contamination events had occurred independently in two different vaccines batches. Indeed, considering how viruses were handled in different laboratories around the world a couple of decades ago, such a mix-up may have happened. During those days, there were neither SOPs nor stringent disinfection protocols implemented when working with viruses. Contamination was likely when working with different viruses or strains of a particular virus at the same time, and thus exchange of viral strains was very common and not restricted in the way it is today. Newer molecular methods, such NGS, uncovered these "misguided" strains all over the world, including in unlikely hosts. Unfortunately, the original CMLV CaPV298-2 sequence is currently unknown, and evidenced-based conclusions cannot be drawn. In this regard, it is important to highlight the fact that the CaPV298-2 strain showed, after 106 passages into Vero cells, a similar deletion to that observed in MVA that originated from Chorioallantois Vaccinia virus Ankara (CVA) after continuous serial passages in chicken fibroblast tissue culture [24]. According to this perspective, it is tempting to speculate that contamination with MVA had occurred before the analysis of the 106th passage of the CaPV298-2 strain. This study certainly has one pitfall. Indeed, two deletions of 5195 and $890 \mathrm{nt}$ with respect to the MVA strain showing the highest nt identity along the whole genome were present. The first is located in a portion of the genome encompassing the ITRs and the left terminal region, the second in the central conserved region. To date, we do not know if these deletions are "real" deletions or merely the evidence of a lack of sequence reads in those portions of the genome. Accordingly, further analyses are currently ongoing. Overall, despite what has been declared in the label, genome sequences of MVA, and not CMLV, were detected in a commercially available CMLV vaccine. However, it is also important to underline that, because all OPXVs are antigenically related, immunization with nearly any OPXV can protect against challenge with other OPXVs [25]. In fact, in the field, this Ducapox vaccine, containing MVA in place of CMLV, has been proven to be capable of protecting animals against CMLV field infection [26,27].

Author Contributions: Conceptualization, M.M., A.I.K. and A.L.; methodology, F.V., Z.M.A.H., M.F.Y., S.M.A.Y. and M.A.A.; software, I.M.; formal analysis, I.M., A.L. and N.D.; investigation, M.M. and A.I.K.; data curation, F.M., C.C. and A.L.; writing-original draft preparation, M.M. and A.I.K.; supervision, A.L., N.D., S.S.A. and G.S.; project administration, S.S.A. and G.S. All authors have read and agreed to the published version of the manuscript.

Funding: This research was funded by a twining project between Istituti Zooprofilattici Sperimentali (IZS) in Italy and Abu Dhabi Agriculture and Food Safety Authority (ADAFSA) in the United Arab Emirates.

Acknowledgments: Mention of trade names or commercial products in this article is solely for the purpose of providing specific information and does not imply recommendation or endorsement by the IZSAM.

Conflicts of Interest: The authors declare no conflict of interest.

\section{References}

1. Bera, B.C.; Riyesh, T.; Barua, S.; Singh, R.K. Camelpox Virus. In Recent Advances in Animal Virology; Malik, Y., Singh, R., Yadav, M., Eds.; Springer: Singapore, 2019; pp. 121-141. [CrossRef]

2. Antoine, G.; Scheiflinger, F.; Dorner, F.; Falkner, F.G. The Complete Genomic Sequence of the Modified Vaccinia Ankara Strain: Comparison with Other Orthopoxviruses. Virology 1998, 244, 365-396. [CrossRef] [PubMed] 
3. Hendrickson, R.C.; Wang, C.; Hatcher, E.L.; Lefkowitz, E.J. Orthopoxvirus Genome Evolution: The Role of Gene Loss. Viruses 2010, 9, 1933-1967. [CrossRef] [PubMed]

4. Afonso, C.L.; Tulman, E.R.; Lu, Z.; Zsak, L.; Sandybaev, N.T.; Kerembekova, U.Z.; Zaitsev, V.L.; Kutish, G.F.; Rock, D.L. The Genome of Camelpox Virus. Virology 2002, 1, 1-9. [CrossRef] [PubMed]

5. Volz, A.; Sutter, G. Modified Vaccinia Virus Ankara: History, Value in Basic Research, and Current Perspectives for Vaccine Development. Adv. Virus. Res. 2017, 97, 187-243. [CrossRef] [PubMed]

6. Reynolds, M.G.; Guagliardo, S.A.J.; Nakazawa, Y.J.; Doty, J.B.; Mauldin, M.R. Understanding orthopoxvirus host range and evolution: From the enigmatic to the usual suspects. Curr. Opin. Virol. 2018, 28, 108-115. [CrossRef]

7. Khalafalla, A.I.; Mohamed, M.E.H. Clinical and epizootiological features of camelpox in eastern Sudan. J Camel. Pract. 1996, 2, 99-102.

8. Yousif, A.A.; Al-Ali, A.M. A case of mistaken identity? Vaccinia virus in a live camelpox vaccine. Biologicals 2012, 40, 495-498. [CrossRef]

9. Kaaden, O.R.; Walz, A.; Czerny, C.P.; Wernery, U. Progress in the development of a camel pox vaccine. In Proceedings of the First International Camel Conference, Dubai, UAE, 2-6 February 1992; pp. 47-49.

10. Khalafalla, A.I.; Abdelazim, F. Human and Dromedary Camel Infection with Camelpox Virus in Eastern Sudan. Vector Borne Zoonotic Dis. 2017, 17, 281-284. [CrossRef]

11. Herrlick, A.; Mayr, A.; Mahnel, H.; Munz, E. Experimental Studies on Transformation of the Variola Virus into the Vaccinia Virus. Archiv für die gesamte Virusforschung. 1963, 12, 579-599. [CrossRef]

12. Pizzurro, F.; Mangone, I.; Zaccaria, G.; De Luca, E.; Malatesta, D.; Innocenti, M.; Carmine, I.; Cito, F.; Marcacci, M.; Di Sabatino, D.; et al. Whole-Genome Sequence of a Suid Herpesvirus-1 Strain Isolated from the Brain of a Hunting Dog in Italy. Genome Announc. 2016, 4, 6. [CrossRef]

13. Emerson, G.L.; Li, Y.; Frace, M.A.; Olsen-Rasmussen, M.A.; Khristova, M.L.; Govil, D.; Sammons, S.A.; Regnery, R.L.; Karem, K.L.; Damon, I.K.; et al. The phylogenetics and ecology of the orthopoxviruses endemic to North America. PLoS ONE 2009, 4, e7666. [CrossRef] [PubMed]

14. Lanave, G.; Dowgier, G.; Decaro, N.; Albanese, F.; Brogi, E.; Parisi, A.; Losurdo, M.; Lavazza, A.; Martella, V.; Buonavoglia, C.; et al. Novel Orthopoxvirus and Lethal Disease in Cat, Italy. Emerg. Infect. Dis. 2018, 24, 1665-1673. [CrossRef] [PubMed]

15. Katoh, K.; Misawa, K.; Kuma, K.; Miyata, T. MAFFT: A novel fast Fourier transform. Nucleic Acids Res. 2002, 30, 3059-3066. [CrossRef]

16. Huelsenbeck, J.P.; Ronquist, F. MRBAYES: Bayesian inference of phylogenetic trees. Bioinformatics 2001, 17, 754-755. [CrossRef] [PubMed]

17. Ronquist, F.; Huelsenbeck, J.P. MrBayes 3: Bayesian phylogenetic inference under mixed models. Bioinformatics 2003, 19, 1572-1574. [CrossRef] [PubMed]

18. Andrews, S. FastQC: A Quality Control Tool for High Throughput Sequence Data. 2010. Available online: http://www.bioinformatics.babraham.ac.uk/projects/fastqc (accessed on 22 July 2020).

19. Bolger, A.M.; Lohse, M.; Usadel, B. Trimmomatic: A flexible trimmer for Illumina Sequence Data. Bioinformatics 2014, 30, 2114-2120. [CrossRef] [PubMed]

20. Langmead, B.; Salzberg, S. Fast gapped-read alignment with Bowtie 2. Nat. Methods 2012, 9, 357-359. [CrossRef]

21. Nurk, S.; Bankevich, A.; Antipov, D.; Gurevich, A.A.; Korobeynikov, A.; Lapidus, A.; Prjibelski, A.D.; Pyshkin, A.; Sirotkin, A.; Sirotkin, Y.; et al. Assembling single-cell genomes and mini-metagenomes from chimeric MDA products. J. Comput. Biol. 2013, 20, 714-737. [CrossRef]

22. Bankevich, A.; Nurk, S.; Antipov, D.; Gurevich, A.A.; Dvorkin, M.; Kulikov, A.S.; Lesin, V.M.; Nikolenko, S.I.; Pham, S.; Prjibelski, A.D.; et al. SPAdes: A New Genome Assembly Algorithm and Its Applications to Single-Cell Sequencing. J. Comput. Biol. 2012, 19, 455-477. [CrossRef]

23. Torsten, S. Prokka: Rapid Prokaryotic Genome Annotation. Boinformatics 2014, 14, 2068-2069. [CrossRef]

24. Pfeffer, M.; Meyer, H.; Wernery, U.; Kaaden, O.R. Comparison of camelpox viruses isolated in Dubai. Vet. Microbiol. 1996, 49, 135-146. [CrossRef]

25. Pauli, G.; Blümel, J.; Burger, R.; Drosten, C.; Gröner, A.; Gürtler, L.; Heiden, M.; Hildebrandt, M.; Jansen, B.; Montag-Lessing, T.; et al. Orthopox Viruses: Infections in Humans. Transfus. Med. Hemother. 2010, 37, 351-364. [CrossRef] [PubMed] 
26. Hafez, S.M.; Al-Sukayran, A.; Dela-Cruz, D.M.; Mazloum, K.S.; Al-Bokmy, A.M.; Al-Mukayel, A.; Amjad, A.M. Development of a live cell culture camelpox vaccine. Vaccine 1992, 10, 533-537. [CrossRef]

27. Wernery, U.; Zachariah, R. Experimental camelpox infection in vaccinated and unvaccinated dromedaries. Zentralbl Veterinarmed B 1999, 46, 131-135. [CrossRef] [PubMed]

(C) 2020 by the authors. Licensee MDPI, Basel, Switzerland. This article is an open access article distributed under the terms and conditions of the Creative Commons Attribution (CC BY) license (http://creativecommons.org/licenses/by/4.0/). 